\title{
Protection of the DIII-D Vessel, Diagnostics and Windows from Microwaves Injected by the ECH/ECCD System
}

\author{
Mirela Cengher ${ }^{1}$, John Lohr $^{1}$, Yuri Gorelov ${ }^{1}$, Antonio Torrezan ${ }^{1}$, Dan Ponce $^{1}$, Xi Chen ${ }^{1}$ \\ ${ }^{1}$ General Atomics, 3550 General Atomics Ct. San Diego, California, USA
}

\begin{abstract}
The ECH/ECCD system on DIII-D benefits from protective measures set in place in order to reduce the likelihood of refracted rf power potentially reaching sensitive areas of the vacuum vessel or diagnostics. In addition to installation of shutters and valves, a density interlock prevents the rf injection into overdense plasma. Visible light cameras, light detectors, Langmuir probes and reflected power sniffers complete the array of diagnostics used for EC protection. Besides the $110 \mathrm{GHz}$ gyrotron, a new higher frequency gyrotron at $117.5 \mathrm{GHz}$ was added to the system during the 2018 experimental campaign. The new frequency allows for operation at higher plasma density and with better current drive efficiency at higher magnetic field than for $110 \mathrm{GHz}$. Injection in O-mode was used in some of the high density plasmas where the $\mathrm{X}$-mode is refracted.
\end{abstract}

\section{Introduction}

Recent years have seen an increase in the large variety of experiments involving injection of $110 \mathrm{GHz}$ EC power into the DIII-D tokamak plasma, with operational regimes approaching or exceeding the density limits for X-mode second harmonic resonance absorption. In these high-density plasmas, the EC beam can be refracted, possibly causing damage to EC launchers or diagnostic systems located behind hightransmission windows. DIII-D has a large array of protections to reduce the possibility of damage. In addition to operational limits for the line averaged plasma density, an array of diagnostics is used for EC protection, including visible light cameras which monitor the light in the launchers, light detectors, and reflected power sniffers, and Langmuir probes. Most recently, a new gyrotron operating at a higher frequency of $117.5 \mathrm{GHz}$ has been added alongside the other operational $110 \mathrm{GHz}, 1.0 \mathrm{MW}$ class gyrotrons in the existing electron cyclotron heating and current drive (ECH/ECCD) complex on the DIII-D tokamak [1], all manufactured at Communications and Power Industries (CPI) [2] has added. The new frequency has a wider operating space in terms of plasma density and is more suitable for high density and higher field plasma, and with higher efficiency of current drive. O-mode second harmonic resonance electron cyclotron heating scenario was used successfully in high density plasmas.

\section{EC beam refraction detection and protection}

Protective measures are in place on DIII-D to reduce the likelihood of refracted $\mathrm{rf}$ power potentially reaching sensitive areas of the vacuum vessel or diagnostics. Ports at risk from an EC beam hit are fitted with shutters, isolation valves, filters, and low loss tangent windows are installed where necessary. Shutters and valves can pre-emptively isolate these sensitive ports during shots when the EC is injected in a plasma close to the overdense conditions that could result in unintentional $\mathrm{rf}$ beam refraction that could cause arcing and damage. In addition, rf filters reject the $110 \mathrm{GHz}$ frequency from the EC system, so the millimeter waves at this frequency can not reach the sensitive electronics dedicated to other spectral ranges [3].

The transmission lines and launchers have the following detection systems installed: rf reflected power monitors acting as sniffers, CCD cameras, visible light monitors, waveguide temperature monitors and Langmuir probes. The Langmuir probes and temperature monitors detect plasma, arcs, or high EC power absorption in the launcher and can remove the EC permit and stop the EC injection if the launcher is at risk. The Langmuir probes are not always reliable since they have also responded to edge localized modes, disruptions, pellet injection, or instabilities. The CCD cameras are monitoring the launcher mirror area without an interlock, providing realtime information about areas of high luminosity or afterglow. The rf injection and absorption for the shots

Corresponding author: cengher@fusion.gat.com 
with potential hot areas identified in the video images are carefully analyzed to eliminate the possibility of power refraction to the EC launchers. The visible light detectors also monitor the general launcher area and have a similar role as the video cameras, the difference being that their signal can be used as an interlock if necessary, without having any details about the areas where the light signal originates. The launcher waveguide temperature is also monitored with two types of detectors for anomalous temperature spikes that could indicate damage to the metal.

Reflected power monitors used as sniffers are installed in each transmission line at the last miter bend before the tokamak. These 4-port rf power monitors are sensitive to the transmission direction, wave polarization, and $\mathrm{rf}$ mode content [4]. A mirror with vacuum on both sides picks off the forward and reflected signals and is followed by a window. An orthomode transducer is then used to measure polarization or mode purity of the injected $\mathrm{rf}$ power. The sniffers respond only to the beam being refracted to the launcher port area of the vessel wall, including the case when the rf beam is launched from another port.

\section{Interlocks and recovery}

To further increase rf protection, the ECH group has implemented a complex set of diagnostics and interlocks. A combination of interlocks and a fault recovery system ensures the rf generation is stopped for the duration of low EC power absorption into the plasma and resumed when absorption conditions are optimal.

Interlock signals are sent by the Plasma Control System (PCS) using feedback from a number of diagnostics. The ECH injection into the tokamak is terminated under command from the PCS when the plasma parameters do not satisfy the pre-set interlock range.

\subsection{Plasma density interlock}

A real-time density interlock is implemented to stop the $\mathrm{ECH}$ if the density rises above a threshold calculated based on the plasma equilibrium reconstruction and electron temperature and density profile measurements. The interlock value is based on ray-tracing model calculations [5] and takes into account the amount of $\mathrm{rf}$ power expected to be absorbed into the plasma and the strike point of the refracted beam in case of low absorption.

When plasma becomes overdense for the injected EC beam, the density interlock stops rf generation, preventing beam refraction that could potentially cause damage to diagnostics or to the ECH launchers. The PCS uses the line-averaged electron density calculated in realtime and compares it to the threshold value, then sends a command to the ECH control system when the line average density reaches a value above the pre-set value. Above this plasma density the X-mode ECH beams would encounter the right hand cutoff before the resonance and would be refracted. The density interlock threshold is adjusted as needed based on ray-tracing of $\mathrm{rf}$ beam propagation at $110 \mathrm{GHz}$ and $117.5 \mathrm{GHz}$.

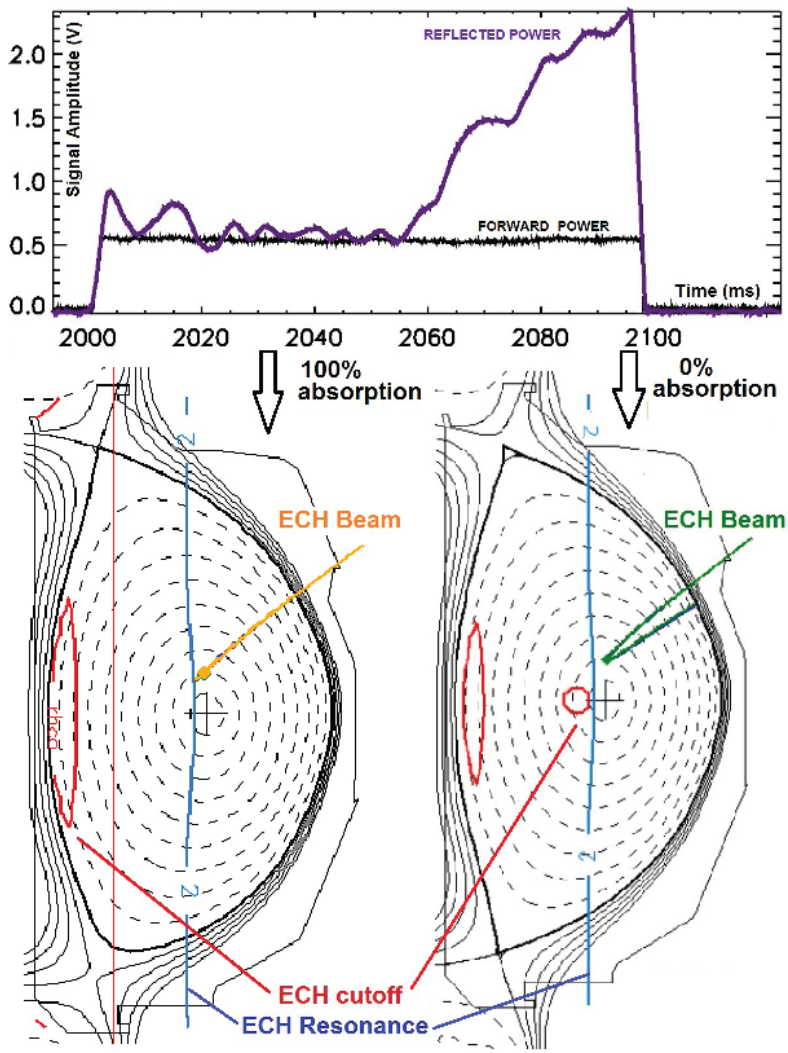

Fig.1. Ray tracing code location of the beam strike point is confirmed by the sniffer response. The sniffer signal (upper plot) shows increasing response as the ECH beam trajectory reaches back to the launcher in the lower right equilibrium reconstruction plot.

An example of the ray tracing of the $\mathrm{ECH}$ beam trajectories for such case is shown in Fig.1. The reflected power monitor response in the upper plot shows increasing response as the ECH beam starts to be refracted. The reconstructed plasma equilibrium and the beam trajectory in the lower right shows the refracted $\mathrm{rf}$ beam near the launch point monitored by the sniffer.

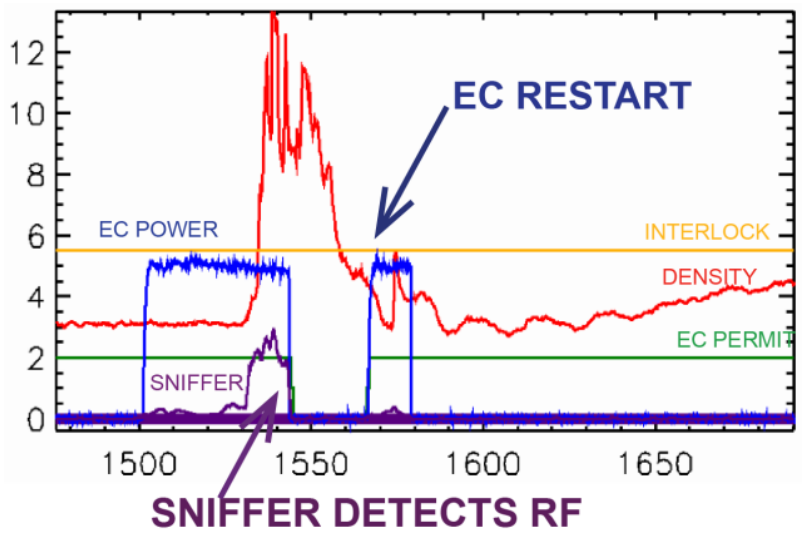

Fig. 2. Time evolution of a gyrotron shot with recovery during ECH injection showing the relevant signals. 
Fig. 2 shows a case where the plasma density increased above the threshold and the interlock was engaged, stopping the $\mathrm{rf}$ generation. At the same time with the beginning of the ramp up of the reflected power, the line-average density exceeded the interlock limit and the EC control system stopped $\mathrm{rf}$ generation. The EC permit was lost with an $8 \mathrm{~ms}$ delay during which the sniffer detected the power refracted from the plasma. The rf generation was resumed once the density interlock is satisfied again.

A number of factors can influence the quality of beam trajectory tracing and the error in the density threshold calculation: the error bars in the density profile measurement and density profile fits, the precision of the aim of the ECH launchers, possible obstruction of the beam by the port boundary, the injected beam polarization, or the fact that strong refraction on density spikes is not considered in the model. The present $8 \mathrm{~ms}$ delay in response to interlock allows rf power to be refracted back to the launcher as seen in Fig. 2 and could end up causing damage even for these short duration periods. Work is under way to reduce this delay in the interlock response to less than $1 \mathrm{~ms}$.

The ECH power is injected into the tokamak from the low field side using four dual launchers [6] and the RF beams can be steered $\pm 20^{\circ}$ in real time during one plasma shot. For the cases when mirrors are moving, the full range of motion is taken into consideration to determine the density interlock value, as the absorption condition can vary widely for a poloidal scan.

\subsection{EC shot restart}

After the interlock is engaged, the rf generation can be resumed later during the same shot if absorption conditions are satisfied, due to a newly added "restart" gyrotron control system feature, so the plasma shot can get full benefit of the ECH/ECCD injection. The new Real-Time Interruptible High-Resolution Control Waveform Generator [7] has made possible the recovery and restart of a gyrotron after a fault, as shown in Fig. 2. Previously, exceeding pre-determined operational limits, or exhibiting off-normal operation, would result in terminating the operation of the faulted gyrotron during that whole plasma shot.

\subsection{Other interlocks}

Other interlocks were implemented to stop EC injection when absorption is expected to be low or absent: plasma current, toroidal magnetic field, plasma early termination due to disruptions. These interlocks offer protection by stopping millimeter wave generation for scenarios such as when no plasma is present, or when the 2 nd harmonic resonance layer is not in the plasma and the absorption at the 3rd harmonic resonance is low. The EC injection into the empty tokamak vessel is allowed for no longer than $250 \mathrm{~ms}$ to protect centerpost tiles and to prevent damage from the beam being reflected on the centerpost and then reaching a high risk port. If the plasma current in the first
$250 \mathrm{~ms}$ is lower than a preset value one of the permits generated by the PCS is lost and interlock stops $\mathrm{rf}$ generation. The plasma current interlock as "no plasma" indicator is used to stop rf generation if the plasma shot is shorter than the requested $\mathrm{EC}$ pulse.

The real-time toroidal magnetic field $\mathrm{B}_{\mathrm{t}}$ interlock stops $\mathrm{rf}$ generation if $\mathrm{B}_{\mathrm{t}}$ drops below the value where the 2nd resonance is no longer in the plasma and can not be reached by the EC beam.

The launcher control system has interlocks that signal that the beam aiming request would be obstructed by the port boundary and the mirror movement is stopped when these limits are reached.

\section{Expanded range of plasma regimes accessible for ECH/ECCD}

For some launch configurations and high density plasmas the $110 \mathrm{GHz} \mathrm{X}$-mode EC beam has low or no absorption. For some of these cases the risk was mitigated by launching an O-mode polarized wave instead of the X-mode, as shown in Fig. $3 \mathrm{~A}$ on the left. The new $117.5 \mathrm{GHz}$ frequency allows the $\mathrm{X}$-mode propagation and absorption for cases where a $110 \mathrm{GHz}$ beam would be refracted, as shown in Fig. $3 \mathrm{~B}$ on the right.

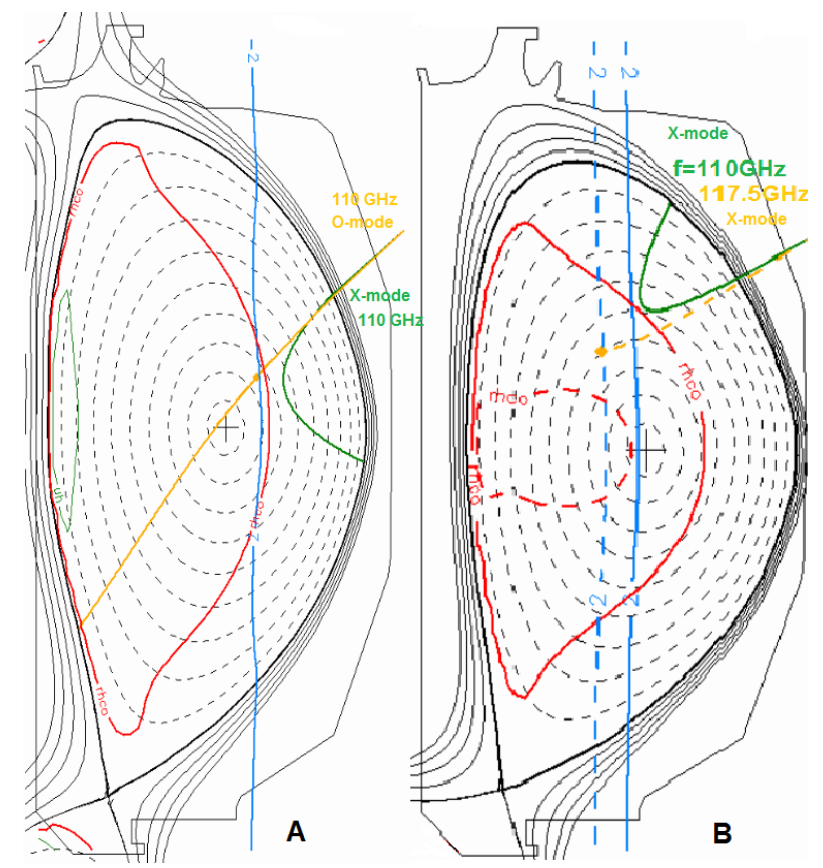

Fig. 3. Equilibrium reconstruction and ray tracing for: A. Xmode and O-mode (yellow) at $110 \mathrm{GHz}$ (green), and B. X mode for $110 \mathrm{GHz}$ (green) and $117.5 \mathrm{GHz}$ (dashed yellow). The right-hand cutoff layer for the X-mode is shown in red. The resonance position is plotted in blue. Solid contours are used for $110 \mathrm{GHz}$ and dashed contours for $117.5 \mathrm{GHz}$.

\subsection{O-mode launch}

O-mode second harmonic resonance plasma heating was used successfully for some of the cases where X-mode cutoff would have no power deposition in the plasma. 
Two grooved mirror polarizers in the miter bends are used to control the elliptical polarization [8]. Polarizers can adjust polarization between shots to change between second harmonic $\mathrm{O}$-mode and $\mathrm{X}$-mode launch.

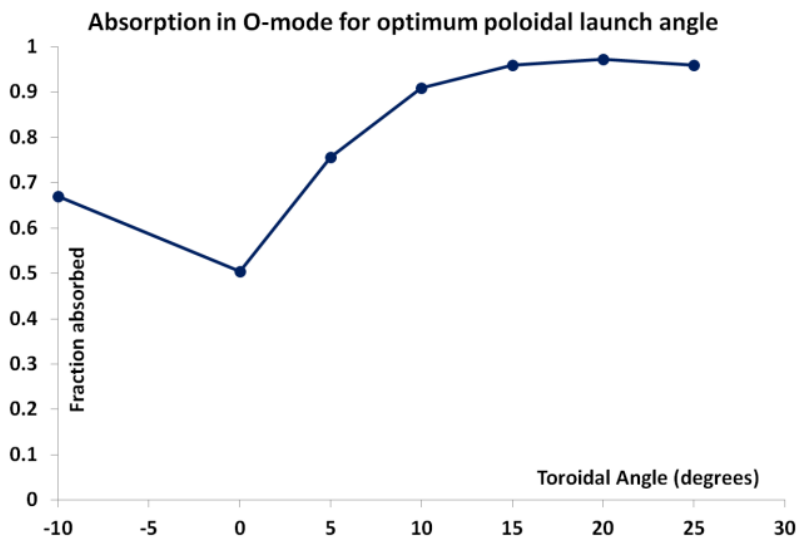

Fig. 4. Fraction of the O-mode power absorbed in the plasma versus the oblique launch angle for the optimum perpendicular launch angle.

O-mode launch has a number of drawbacks: incomplete absorption in the first pass, must be launched at an oblique angle for optimal heating power deposition (as shown in Fig. 4), cannot be used for current drive, and has a wide deposition profile. The advantage is the much higher density cutoff compared to the X-mode. Aiming was set to ensure absorption in the first pass, and also in the second pass after reflection on centerpost if possible and to avoiding hitting diagnostics on the vessel wall. O-mode can be used in limited situations because of limited range of plasmas where absorption in this scenario is high enough, above $80 \%$, and because it cannot be used for current drive due to very low efficiency.

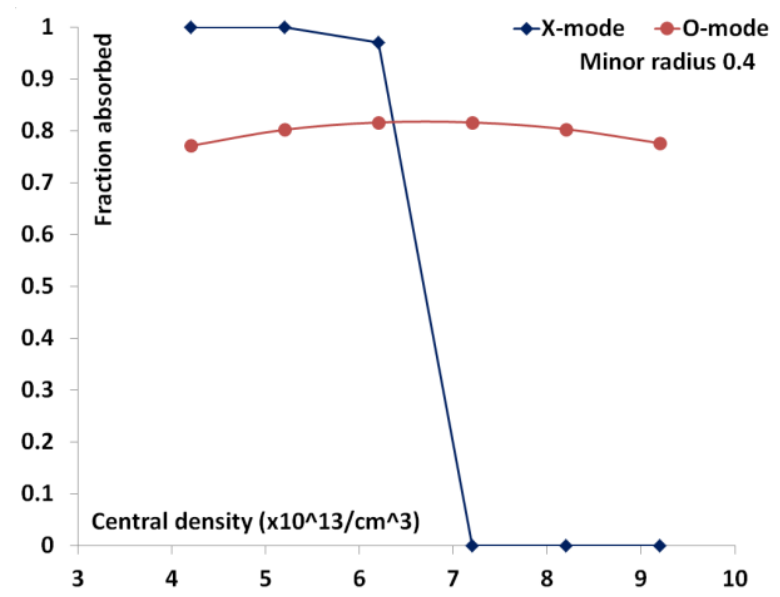

Fig. 5. Fraction of the O-mode and X-mode power absorbed in a single pass in the plasma versus the central electron plasma density for peak power deposition at normalized minor radius $\rho=0.4$.

The fraction of $\mathrm{rf}$ power absorbed in X-mode and Omode for EC heating was investigated using TORAY ray-tracing. Fig. 5 shows the fraction of power absorbed at $\rho=0.4$ versus the central electron density. The full power launched in X-mode is absorbed only at low densities since the $\mathrm{rf}$ has access to the 2nd harmonic resonance. At electron densities above $6.5 \times 10^{13} \mathrm{~cm}^{-3}$ the right-hand cutoff layer is encountered before the resonance, thus leading to an abrupt drop in the fraction of power absorbed in the plasma. The O-mode absorption is around $80 \%$ in the first pass for the whole range investigated, so it can still be used (less efficiently) in some cases where the interlock would stop the Xmode launching into the plasma. A second pass could lead to extra absorption for the O-mode if the beam reflected from the centerpost is in the right mode, and is directed so it encounters the resonance.

\subsection{Higher frequency X-mode launch}

A new design depressed collector gyrotron operating at $117.5 \mathrm{GHz}$ was used in the 2018 experimental campaign. The new frequency allows for rf injection into higher line-averaged plasma density, while providing a comparable efficiency for current drive, and better access to the resonance at higher magnetic field than the other frequency used in the system, $110 \mathrm{GHz}$.

Fig. 3 B shows a case where launched $117.5 \mathrm{GHz}$ is absorbed in the plasma while $110 \mathrm{GHz}$ beams are refracted due to different positions of the right-hand cutoff layers for the two frequencies. The $110 \mathrm{GHz}$ power launched at larger steering angles (less central) would still be absorbed.

\section{Summary}

The DIII-D ECH system features a flexible versatile protection system that includes shutters, valves, windows, filters, cameras, light detectors, Langmuir probes, and rf monitors. The interlock protection has been extensively used and, as a result, rf injection for high risk conditions was avoided. The range of plasma regimes that can operate with EC power injection was extended by using second harmonic resonance $\mathrm{O}$-mode injection and a new slightly higher frequency for the $\mathrm{X}$ mode case. The EC shot recovery feature is a recent upgrade that increased reliability for shots with interlock interruptions.

\section{Acknowledgments}

This work was supported by the U.S. Department of Energy under DE-FC02-04ER54698.

DIII-D data shown in this paper can be obtained in digital format by following the links at https://fusion.gat.com/global/D3D DMP.

Disclaimer: This report was prepared as an account of work sponsored by an agency of the United States Government. Neither the United States Government nor any agency thereof, nor any of their employees, makes any warranty, express or implied, or assumes any legal liability or responsibility for the accuracy, completeness, or usefulness of any information, apparatus, product, or 
process disclosed, or represents that its use would not infringe privately owned rights. Reference herein to any specific commercial product, process, or service by trade name, trademark, manufacturer, or otherwise does not necessarily constitute or imply its endorsement, recommendation, or favoring by the United States Government or any agency thereof. The views and opinions of authors expressed herein do not necessarily state or reflect those of the United States Government or any agency thereof.

\section{References}

[1] M. Cengher, et al., IEEE Transactions on Plasma Science, vol. PP, 99 (2016), pp.1-6

[2] K.Felch, M.Blank, P.Borchard and S. Cauffman, EPJ Web of Conferences, 87, 04006 (2015)

[3] J. Lohr, et al., EPJ Web of Conferences 147, 03003 (2017), pp1-6

[4] M.Cengher, et al, IEEE Transactions on Plasma Science, 42, 7 (2014), pp.1964-1970

[5] K.Matsuda,IEEE Transactions on Plasma Science, $17 \quad 1$ (1989),p.6-11

[6] R.Ellis, et al., Proc.14thTopical Conf. on RF Power in Plasma, AIP Conf. Proc., 595 (2011), pp. 318-321.

[7] D. Ponce et al., Fusion Science snd Technology, 73 (2018), pp 1.

[8] J. Doane, Int.J. Infrared Millimeter Waves 13 (1992), p.1727 